\title{
Omnibus tests for multivariate normality based on Mardia's skewness and kurtosis using normalizing transformation
}

\author{
Namhyun $\mathrm{Kim}^{1, a}$ \\ ${ }^{a}$ Department of Science, Hongik University, Korea
}

\begin{abstract}
Mardia (Biometrika, 57, 519-530, 1970) defined measures of multivariate skewness and kurtosis. Based on these measures, omnibus test statistics of multivariate normality are proposed using normalizing transformations. The transformations we consider are normal approximation and a Wilson-Hilferty transformation. The normalizing transformation proposed by Enomoto et al. (Communications in Statistics-Simulation and Computation, 49, 684-698, 2019) for the Mardia's kurtosis is also considered. A comparison of power is conducted by a simulation study. As a result, sum of squares of the normal approximation to the Mardia's skewness and the Enomoto's normalizing transformation to the Mardia's kurtosis seems to have relatively good power over the alternatives that are considered.
\end{abstract}

Keywords: goodness-of-fit test, Mardia's kurtosis, Mardia's skewness, multivariate normality, power comparison

\section{Introduction}

Assessing the assumption of multivariate normality is an important issue in statistical research, especially in classical multivariate analysis. A vast number of test procedures for multivariate normality are found in the literature. For a general review, some references are Henze and Zirkler (1990), Henze (2002), Thode (2002, Chapter 9), and Srivastava and Mudholkar (2003). Comparative studies in power are done in Horswell and Looney (1992), Romeu and Ozturk (1993), Mecklin and Mundfrom (2005), Farrell et al. (2007), and Hanusz et al. (2018).

The Henze and Zirkler (1990) test is sometimes recommended as a formal test for multinormality because its invariance and consistency are proven theoretically and it has relatively good power across a wide range of alternatives (Mecklin and Mundfrom, 2005; Farrell et al., 2007). However we also need some supplementary or less formal procedures to follow up such as some graphical procedures to diagnose possible deviations from normality.

The classical Jarque and Bera (1980) test is a popular test for univariate normality. The test is the sum of squares of the standardized univariate skewness and kurtosis using their asymptotic mean and variance, which is asymptotically distributed as a $\chi^{2}$-variate. It is also known as the D'Agostino and Pearson (1973) or the Bowman and Shenton (1975) test in Statistics. The Jarque and Bera test is emphasized especially in Econometrics and its modifications are proposed by Urzua (1996), Gel and Gastwirth (2008), and Stehlík et al. (2012).

\footnotetext{
${ }^{1}$ Department of Science, Hongik University, 94 Wausan-ro, Mapo-gu, Seoul 04066, Korea. E-mail: nhkim@ @ongik.ac.kr

Published 30 September 2020 / journal homepage: http://csam.or.kr

(c) 2020 The Korean Statistical Society, and Korean International Statistical Society. All rights reserved.
} 
D'Agostino and Pearson $(1973,1974)$ proposed sum of transformed skewness and kurtosis to test univariate normality. They used the transformation of D'Agostino (1970) for skewness and the Anscombe and Glynn (1983)'s transformation for kurtosis (Kim, 2016). Doornik and Hansen (2008) also derived a similar type of statistic using a transformation from Shenton and Bowman (1977) to kurtosis. They claimed that their test tried to take care of the small sample dependence between skewness and kurtosis.

Mardia $(1970,1974)$ procedures are a common test for multivariate normality in regard to skewness or kurtosis. The skewness or kurtosis approach can provide direct measure of departure from normality with strong point over other procedures. Baringhaus and Henze (1992) studied the limit distribution of the Mardia's skewness under some alternatives. Henze (1994) obtained the limiting non-null distribution of Mardia's kurtosis.

Mardia and Foster (1983) also considered statistics of sum of the transformed multivariate skewness and kurtosis. In this paper, we investigate a multivariate version of the Jarque-Bera type test based on the Mardia's skewness and kurtosis, and statistics of sum of the transformed multivariate skewness and kurtosis like done in Mardia and Foster (1983) using some normalizing transformations. Section 2 describes the transformations we use and presents test statistics for multivariate normality. Section 3 contains a comparison of power performances of the test statistics through a simulation study. Section 4 ends the paper with concluding remarks.

\section{Test statistics}

Let $\mathbf{X}_{1}, \ldots, \mathbf{X}_{n}$ be a random sample of size $n$ from a $p$-dimensional population, and let $N_{p}(\mu, \boldsymbol{\Sigma})$ be a $p$-variate multivariate normal distribution with mean vector $\mu$ and covariance matrix $\boldsymbol{\Sigma}$. We want to test the null hypothesis

$$
H_{0}: \mathbf{X}_{1}, \ldots, \mathbf{X}_{n} \text { is a sample from } N_{p}(\mu, \boldsymbol{\Sigma}) \text { for some } \mu \text { and } \boldsymbol{\Sigma} .
$$

Let

$$
\overline{\boldsymbol{X}}=\frac{1}{n} \sum_{j=1}^{n} \boldsymbol{X}_{j}, \quad \boldsymbol{S}=\frac{1}{n} \sum_{j=1}^{n}\left(\boldsymbol{X}_{j}-\overline{\boldsymbol{X}}\right)\left(\boldsymbol{X}_{j}-\overline{\boldsymbol{X}}\right)^{\prime},
$$

be a sample mean vector and a sample covariance matrix, respectively.

According to Mardia $(1970,1974)$, the multivariate measure of skewness $b_{1, p}$ and kurtosis $b_{2, p}$ are defined as

$$
b_{1, p}=\frac{1}{n^{2}} \sum_{i=1}^{n} \sum_{j=1}^{n}\left\{\left(\boldsymbol{X}_{i}-\overline{\boldsymbol{X}}\right)^{\prime} \boldsymbol{S}^{-1}\left(\boldsymbol{X}_{j}-\overline{\boldsymbol{X}}\right)\right\}^{3} \text {, }
$$

and

$$
b_{2, p}=\frac{1}{n} \sum_{j=1}^{n}\left\{\left(X_{j}-\bar{X}\right)^{\prime} S^{-1}\left(X_{j}-\bar{X}\right)\right\}^{2}
$$

where $\overline{\boldsymbol{X}}$ and $\boldsymbol{S}$ are defined in (2.1). Mardia (1970) showed

$$
M_{s}\left(b_{1, p}\right)=\frac{n b_{1, p}}{6} \stackrel{d}{\rightarrow} \chi^{2}\left(\frac{p(p+1)(p+2)}{6}\right)
$$


and

$$
\frac{n\left(b_{2, p}-p(p+2)\right)^{2}}{8 p(p+2)} \stackrel{d}{\rightarrow} \chi^{2}(1) .
$$

Using the above asymptotic result, Mardia and Foster (1983) considered the normal approximation of a $\chi^{2}$ variable for the skewness $b_{1, p}, N_{s}\left(b_{1, p}\right)$,

$$
N_{s}\left(b_{1, p}\right)=\frac{b_{1, p}-\frac{6 f}{n}}{\frac{6}{n} \sqrt{2 f}}
$$

with $f=p(p+1)(p+2) / 6$, and the Wilson-Hilferty transformation of a $\chi^{2}$ variable, $W_{s}\left(b_{1, p}\right)$

$$
W_{s}\left(b_{1, p}\right)=\frac{\left(\frac{n}{6} \frac{b_{1, p}}{f}\right)^{\frac{1}{3}}-\left(1-\frac{2}{9 f}\right)}{\sqrt{\frac{2}{9 f}}} .
$$

By the asymptotic result in (2.5), we may consider the normal approximation of the kurtosis $b_{2, p}$,

$$
T_{k}\left(b_{2, p}\right)=\frac{b_{2, p}-p(p+2)}{\sqrt{\frac{8 p(p+2)}{n}}} .
$$

By the way, Mardia $(1970,1974)$ and Mardia and Kanazawa (1983) derived the exact mean and variance of $b_{2, p}$

$$
\begin{aligned}
E\left(b_{2, p}\right) & =\frac{n-1}{n+1} p(p+2), \\
\operatorname{Var}\left(b_{2, p}\right) & =\frac{(n-3)(n-p-1)(n-p+1)}{(n+1)^{2}(n+3)(n+5)} 8 p(p+2) .
\end{aligned}
$$

Hence we will use

$$
N_{k}\left(b_{2, p}\right)=\frac{b_{2, p}-\frac{n-1}{n+1} p(p+2)}{\sqrt{\frac{(n-3)(n-p-1)(n-p+1)}{(n+1)^{2}(n+3)(n+5)} 8 p(p+2)}}
$$

instead of (2.8). Mardia and Kanazawa (1983), Mardia and Foster (1983) suggested the WilsonHilferty approximation for $b_{2, p}$ such that

$$
W_{k}\left(b_{2, p}\right)=3\left(\frac{f_{1}}{2}\right)^{\frac{1}{2}}\left[1-\frac{2}{9 f_{1}}-\left(\frac{1-\frac{2}{f_{1}}}{1+N_{k}\left(b_{2, p}\right)\left(\frac{2}{f_{1}-4}\right)^{\frac{1}{2}}}\right)^{\frac{1}{3}}\right],
$$

where $N_{k}\left(b_{2, p}\right)$ is defined in $(2.9)$ and $f_{1}$ is

$$
f_{1}=6+\sqrt{n}\left(\frac{8 p(p+2)}{(p+8)^{2}}\right)^{\frac{1}{2}}\left[\frac{\sqrt{n}(p(p+2))^{\frac{1}{2}}}{\sqrt{2}(p+8)}+\left(1+\frac{n p(p+2)}{2(p+8)^{2}}\right)^{\frac{1}{2}}\right] .
$$


They used the null distribution of $b_{2, p}$ may be approximated by the inverse of a $\chi^{2}$ distribution with the above degree of freedom $f_{1}$.

Enomoto et al. (2019) proposed another normal transformation for the kurtosis $b_{2, p}$. The derivation of their normalizing transformation in multivariate analysis is based on Konishi (1981) and Seo et al. (1994). That is

$$
E_{k}\left(b_{2, p}\right)=\sqrt{\frac{n}{8 p(p+2)}}\left[\frac{\exp \left(d\left(b_{2, p}-p(p+2)\right)-1\right)}{d}+\frac{2 p(p+2)(1-2 d)}{n}\right]
$$

with $d=-(p+8) /(3 p(p+2))$.

Based on the multivariate measure defined in (2.2) and (2.3) and the transformations (2.6) to (2.11), we consider test statistics as follows.

$$
\begin{gathered}
M N=M_{s}\left(b_{1, p}\right)+N_{k}^{2}\left(b_{2, p}\right), \\
M W=M_{s}\left(b_{1, p}\right)+W_{k}^{2}\left(b_{2, p}\right), \\
M E=M_{s}\left(b_{1, p}\right)+E_{k}^{2}\left(b_{2, p}\right), \\
N N=N_{s}^{2}\left(b_{1, p}\right)+N_{k}^{2}\left(b_{2, p}\right), \\
N W=N_{s}^{2}\left(b_{1, p}\right)+W_{k}^{2}\left(b_{2, p}\right), \\
N E=N_{s}^{2}\left(b_{1, p}\right)+E_{k}^{2}\left(b_{2, p}\right), \\
W N=W_{s}^{2}\left(b_{1, p}\right)+N_{k}^{2}\left(b_{2, p}\right), \\
W W=W_{s}^{2}\left(b_{1, p}\right)+W_{k}^{2}\left(b_{2, p}\right), \\
W E=W_{s}^{2}\left(b_{1, p}\right)+E_{k}^{2}\left(b_{2, p}\right) .
\end{gathered}
$$

The letters of the statistics come from the name of each transformation to the multivariate skewness and kurtosis $b_{1, p}, b_{2, p}$ in (2.2), (2.3). $N_{s}^{2}\left(b_{1, p}\right), W_{s}^{2}\left(b_{1, p}\right), N_{k}^{2}\left(b_{2, p}\right), W_{k}^{2}\left(b_{2, p}\right)$, and $E_{k}^{2}\left(b_{2, p}\right)$ follow $\chi^{2}(1)$ asymptotically; therefore, $M N, M W, M E$ have the asymptotic distribution $\chi^{2}(p(p+1)(p+2) / 6+1)$ by (2.4), and all the other statistics follow $\chi^{2}(2)$ asymptotically. Because of the covariance between these two statistics, $b_{1, p}, b_{2, p}$, a large sample is required for the statistics to follow the asymptotic distributions.

Doornik and Hansen (2008) considered an omnibus test

$$
M_{p}=\frac{n b_{1, p}}{6}+\frac{n\left(b_{2, p}-p(p+2)\right)^{2}}{8 p(p+2)}
$$

to test the multivariate normality. The statistic has almost the same form as the statistic $M N$.

The Henze and Zirkler (1990) statistic is often recommended as a formal test statistic for multivariate normality. It is

$$
T_{n, \beta}\left(\mathbf{X}_{1}, \ldots, \mathbf{X}_{n}\right)=n\left(4 I(\mathbf{S} \text { is singular })+D_{n, \beta} I(\mathbf{S} \text { is nonsingular })\right),
$$

where

$$
D_{n, \beta}=\frac{1}{n^{2}} \sum_{i, j=1}^{n} \exp \left(-\frac{\beta^{2}}{2}\left\|\mathbf{Y}_{i}-\mathbf{Y}_{j}\right\|^{2}\right)-2\left(1+\beta^{2}\right)^{-\frac{p}{2}} \frac{1}{n} \sum_{j=1}^{n} \exp \left(-\frac{\beta^{2}}{2\left(1+\beta^{2}\right)}\left\|\mathbf{Y}_{j}\right\|^{2}\right)+\left(1+2 \beta^{2}\right)^{-\frac{p}{2}}
$$


Table 1: statistics and $p$-values for the contrasts of Rao's bark deposit data

\begin{tabular}{|c|c|c|c|c|c|c|c|c|c|}
\hline & $M N$ & $M W$ & $M E$ & $N N$ & $N W$ & $N E$ & $W N$ & $W W$ & $W E$ \\
\hline statistics & 5.57 & 5.5 & 5.5 & 1.09 & 1.03 & 1.02 & 1.21 & 1.15 & 1.14 \\
\hline$p$-values & 0.90 & 0.9 & 0.9 & 0.58 & 0.60 & 0.60 & 0.55 & 0.56 & 0.57 \\
\hline
\end{tabular}

with $\left\|\mathbf{Y}_{i}-\mathbf{Y}_{j}\right\|^{2}=\left(\mathbf{X}_{i}-\mathbf{X}_{j}\right)^{\prime} \mathbf{S}^{-1}\left(\mathbf{X}_{i}-\mathbf{X}_{j}\right)$ and $\left\|\mathbf{Y}_{j}\right\|^{2}=\left(\mathbf{X}_{j}-\overline{\mathbf{X}}\right)^{\prime} \mathbf{S}^{-1}\left(\mathbf{X}_{j}-\overline{\mathbf{X}}\right) . \beta$ is defined as

$$
\beta=\beta_{p}(n)=\frac{1}{\sqrt{2}}\left(\frac{2 p+1}{4}\right)^{\frac{1}{p+4}} n^{\frac{1}{p+4}} .
$$

The Henze and Zirkler statistic in (2.13) is also included in the power comparison in Section 3.

\section{Example and power comparison}

\subsection{Example}

The data set in Rao (1948) is considered. It consists of the thickness of bark deposit on 28 cork trees measured by the weight of cork borings from four directions; north $(\mathrm{N})$, east $(\mathrm{E})$, west $(\mathrm{W})$, and south (S). The concern is to investigate if the thickness of bark deposit varies in the four directions. He selected three constraints

$$
Y_{1}=N-E-W+S, \quad Y_{2}=S-W, \quad Y_{3}=N-S
$$

by the reason he explained in the paper and tested $E\left(Y_{i}\right)=0, i=1,2,3$ for this purpose. To test the problem by applying some techniques like Hotelling's $T^{2}$ test, the assumption of multivariate normality should be valid. Pearson (1956) examined the same data set.

For the contrasts $\left(Y_{1}, Y_{2}, Y_{3}\right)$, the statistics in Section 2 and the corresponding $p$-values are presented in Table 1. The $p$-values are computed using $\chi^{2}$ distributions with degree of freedom 11 or 2. The results confirm that Rao's test for contrasts is valid since the multivariate normality of the contrasts cannot be rejected. The Henze-Zirkler statistic for the contrasts is 0.68 , and the critical point for $\alpha=0.1$ in Table 3.2 in Henze and Zirkler (1990) is 0.803 ; therefore the multivariate normality still cannot be rejected. Mardia (1975), Srivastava and Hui (1987), and Kim (2016) also dealt with the data set and have the same conclusion for the contrasts.

\subsection{Power comparison}

We performed a simulation to study the proposed test statistics in Section 2. They are combinations of the transformed Mardia's multivariate skewness and kurtosis. The statistics are suggested as omnibus ones.

Tables 2-5 show the empirical power of the 9 test statistics and the Henze and Zirkler $(H Z)$ statistic in (2.13) at the significance level $\alpha=0.05$ for the combinations of dimensions $p=2,5$ and sample sizes $n=20,50$. We used the critical values from the simulation, not from the asymptotic distributions to correct the size of the tests. We generated $N=10,000$ random samples for the null distributions of the statistics. The samples are generated from $N_{p}(\mathbf{0}, \boldsymbol{I})$ since the distributions of the statistics are invariant on $\boldsymbol{\mu}$ and $\boldsymbol{\Sigma}$. The simulation results for the null distributions are not presented. When we investigate the results, the null of $N N, N E$, and $W E$ statistics are well described by the asymptotic distribution $\chi^{2}(2)$. As for the $H Z$ statistic, we used the critical values given in Table 3.1 and 3.3 in Henze and Zirkler (1990). 
Table 2: power comparison of the statistics $(\alpha=0.05, p=2, n=20)$

\begin{tabular}{|c|c|c|c|c|c|c|c|c|c|c|}
\hline Alternative & $M N$ & $M W$ & $M E$ & $N N$ & $N W$ & $N E$ & $W N$ & $W W$ & $W E$ & $H Z$ \\
\hline$N(0,1)^{2}$ & 0.05 & 0.05 & 0.06 & 0.05 & 0.04 & 0.06 & 0.05 & 0.05 & 0.06 & 0.05 \\
\hline Cauchy $(0,1)^{2}$ & 0.97 & 0.94 & 0.95 & 0.98 & 0.92 & 0.94 & 0.97 & 0.91 & 0.92 & 0.97 \\
\hline $\operatorname{Logistic}(0,1)^{2}$ & 0.17 & 0.14 & 0.17 & 0.17 & 0.10 & 0.14 & 0.15 & 0.08 & 0.11 & 0.10 \\
\hline$\left(t_{2}\right)^{2}$ & 0.74 & 0.67 & 0.71 & 0.74 & 0.61 & 0.67 & 0.71 & 0.56 & 0.61 & 0.64 \\
\hline$\left(t_{5}\right)^{2}$ & 0.30 & 0.24 & 0.30 & 0.30 & 0.18 & 0.25 & 0.26 & 0.15 & 0.19 & 0.17 \\
\hline $\operatorname{Beta}(1,1)^{2}$ & 0.00 & 0.29 & 0.02 & 0.04 & 0.37 & 0.23 & 0.11 & 0.41 & 0.26 & 0.18 \\
\hline $\operatorname{Beta}(2,2)^{2}$ & 0.00 & 0.12 & 0.01 & 0.01 & 0.16 & 0.08 & 0.06 & 0.18 & 0.12 & 0.06 \\
\hline $\operatorname{Beta}(1,2)^{2}$ & 0.04 & 0.11 & 0.06 & 0.04 & 0.13 & 0.09 & 0.04 & 0.12 & 0.06 & $0.28 \dagger$ \\
\hline $\exp (1)^{2}$ & 0.69 & 0.67 & 0.76 & 0.70 & 0.60 & 0.71 & 0.61 & 0.50 & 0.61 & $0.86 \dagger$ \\
\hline $\operatorname{Lognormal}(0,0.5)^{2}$ & 0.50 & 0.47 & 0.56 & 0.50 & 0.41 & 0.51 & 0.43 & 0.34 & 0.42 & $0.58 \dagger$ \\
\hline $\operatorname{Gamma}(0.5,1)^{2}$ & 0.90 & 0.90 & 0.93 & 0.91 & 0.86 & 0.93 & 0.85 & 0.77 & 0.86 & $0.99 \dagger$ \\
\hline $\operatorname{Gamma}(5,1)^{2}$ & 0.21 & 0.20 & 0.26 & 0.21 & 0.15 & 0.22 & 0.17 & 0.12 & 0.14 & 0.24 \\
\hline$\left(\chi_{5}^{2}\right)^{2}$ & 0.37 & 0.34 & 0.42 & 0.37 & 0.28 & 0.38 & 0.30 & 0.20 & 0.29 & $0.45 \dagger$ \\
\hline$\left(\chi_{15}^{2}\right)^{2}$ & 0.16 & 0.15 & 0.19 & 0.15 & 0.11 & 0.16 & 0.14 & 0.09 & 0.12 & 0.16 \\
\hline$N(0,1) * t_{5}$ & 0.17 & 0.13 & 0.16 & 0.17 & 0.11 & 0.15 & 0.14 & 0.09 & 0.11 & 0.12 \\
\hline$N(0,1) * \operatorname{Beta}(1,1)$ & 0.01 & 0.09 & 0.03 & 0.02 & 0.13 & 0.07 & 0.05 & 0.15 & 0.10 & 0.10 \\
\hline$N(0,1) * \exp (1)$ & 0.40 & 0.36 & 0.46 & 0.39 & 0.32 & 0.40 & 0.32 & 0.24 & 0.30 & $0.51 \dagger$ \\
\hline$N(0,1) * \chi_{5}^{2}$ & 0.20 & 0.18 & 0.23 & 0.20 & 0.15 & 0.19 & 0.16 & 0.11 & 0.14 & 0.22 \\
\hline $\operatorname{NMIX}_{2}(0.5,4,0,0)$ & 0.01 & 0.18 & 0.03 & 0.03 & 0.23 & 0.15 & 0.07 & 0.25 & 0.16 & $0.52 \dagger$ \\
\hline $\operatorname{NMIX}_{2}(0.5,0,0,0.9)$ & 0.18 & 0.14 & 0.19 & 0.18 & 0.09 & 0.15 & 0.16 & 0.06 & 0.11 & 0.14 \\
\hline $\operatorname{NMIX}_{2}(0.5,4,0,0.9)$ & 0.23 & 0.25 & 0.34 & 0.25 & 0.20 & 0.27 & 0.16 & 0.13 & 0.16 & $0.82 \dagger$ \\
\hline $\mathrm{NMIX}_{2}(0.9,4,0,0)$ & 0.55 & 0.51 & 0.61 & 0.53 & 0.42 & 0.54 & 0.46 & 0.32 & 0.42 & $0.64 \dagger$ \\
\hline $\operatorname{NMIX}_{2}(0.9,0,0,0.9)$ & 0.06 & 0.05 & 0.08 & 0.06 & 0.05 & 0.06 & 0.06 & 0.04 & 0.05 & 0.05 \\
\hline $\operatorname{NMIX}_{2}(0.9,4,0,0.9)$ & 0.54 & 0.51 & 0.63 & 0.56 & 0.44 & 0.57 & 0.45 & 0.32 & 0.43 & 0.62 \\
\hline
\end{tabular}

$N=5,000$ samples are generated from each of the various alternative distributions. As alternatives, we included the distributions with independent marginals and mixtures of normal distributions. $F_{1} * F_{2}$ denotes the distribution with independent marginal distributions $F_{1}$ and $F_{2} . F_{1}^{p}$ denotes the product of $p$ independent copies of $F_{1}$. $\operatorname{NMIX}_{p}\left(\kappa, \mu, \rho_{1}, \rho_{2}\right)$ stands for the normal mixture

$$
\kappa N_{p}\left(\mathbf{0}, \boldsymbol{R}_{1}\right)+(1-\kappa) N_{p}\left(\mu \mathbf{1}, \boldsymbol{R}_{2}\right),
$$

where $\boldsymbol{R}_{i}$ is a matrix with diagonal elements equal to 1 and off-diagonal equal to $\rho_{i}, 0 \leq \rho_{i}<1$, $i=1,2$.

The power results in Tables $2-5$ indicate the following. The best power among 9 statistics based on the multivariate skewness and kurtosis to 4 places of decimals for each alternative is written in bold to draw a distinction, although they are rounded off to the nearest hundredth in the tables. When the $H Z$ statistic has the best power, the number is marked as a dagger ( $\dagger)$. First, the statistics with the normal approximation to kurtosis, $M N, N N, W N$ show good power against alternatives with symmetric thicker tailed marginals such as Cauchy, logistic, and $t$-distributions. Second, the statistics with the Wilson-Hilferty transformation to kurtosis show better power against alternatives with symmetric shorter tailed marginals $\operatorname{Beta}(1,1), \operatorname{Beta}(2,2)$. However the power of the statistic $M W$ is not good for $p=5$. The statistics $N E, W E$ with the Enomoto transformation to kurtosis also show relatively good power against these alternatives, although the power is lower than $N W, W W$, respectively. $N W$ or $W W$ are recommended to use just for these alternatives, and $W W$ shows a little bit better power than $N W$ against these alternatives. Third, the statistics $M N, M W$, and $M E$ show relatively good power against alternatives with skewed marginals especially for $p=5$. The statistic $M E$ shows the best power for $p=2$, but they show almost the same power for $p=5$. Those statistics $M N, M W$, and $M E$ have good power against the normal mixture alternatives. Fourth, the statistic $H Z$ shows the 
Table 3: power comparison of the statistics ( $\alpha=0.05, p=2, n=50$ )

\begin{tabular}{|c|c|c|c|c|c|c|c|c|c|c|}
\hline Alternative & $M N$ & $M W$ & $M E$ & $N N$ & $N W$ & $N E$ & $W N$ & $W W$ & $W E$ & $H Z$ \\
\hline$N(0,1)^{2}$ & 0.05 & 0.05 & 0.05 & 0.05 & 0.04 & 0.05 & 0.05 & 0.05 & 0.06 & 0.05 \\
\hline Cauchy $(0,1)^{2}$ & 1.00 & 1.00 & 1.00 & 1.00 & 1.00 & 1.00 & 1.00 & 1.00 & 1.00 & $1.00 \dagger$ \\
\hline $\operatorname{Logistic}(0,1)^{2}$ & 0.32 & 0.27 & 0.28 & 0.33 & 0.23 & 0.26 & 0.32 & 0.21 & 0.22 & 0.15 \\
\hline$\left(t_{2}\right)^{2}$ & 0.97 & 0.95 & 0.93 & 0.97 & 0.94 & 0.94 & 0.97 & 0.95 & 0.94 & 0.95 \\
\hline$\left(t_{5}\right)^{2}$ & 0.56 & 0.50 & 0.50 & 0.56 & 0.46 & 0.48 & 0.56 & 0.44 & 0.44 & 0.32 \\
\hline $\operatorname{Beta}(1,1)^{2}$ & 0.00 & 0.76 & 0.48 & 0.30 & 0.86 & 0.81 & 0.42 & 0.90 & 0.82 & 0.68 \\
\hline $\operatorname{Beta}(2,2)^{2}$ & 0.00 & 0.23 & 0.07 & 0.03 & 0.36 & 0.31 & 0.13 & 0.44 & 0.34 & 0.17 \\
\hline $\operatorname{Beta}(1,2)^{2}$ & 0.12 & 0.30 & 0.28 & 0.13 & 0.26 & 0.29 & 0.05 & 0.21 & 0.16 & $0.80 \dagger$ \\
\hline $\exp (1)^{2}$ & 1.00 & 1.00 & 1.00 & 1.00 & 1.00 & 1.00 & 0.99 & 0.99 & 0.99 & 1.00 \\
\hline $\operatorname{Lognormal}(0,0.5)^{2}$ & 0.95 & 0.95 & 0.96 & 0.94 & 0.94 & 0.95 & 0.92 & 0.88 & 0.91 & 0.94 \\
\hline $\operatorname{Gamma}(0.5,1)^{2}$ & 1.00 & 1.00 & 1.00 & 1.00 & 1.00 & 1.00 & 1.00 & 1.00 & 1.00 & $1.00 \dagger$ \\
\hline $\operatorname{Gamma}(5,1)^{2}$ & 0.56 & 0.58 & 0.63 & 0.57 & 0.56 & 0.61 & 0.47 & 0.46 & 0.48 & 0.53 \\
\hline$\left(\chi_{5}^{2}\right)^{2}$ & 0.86 & 0.86 & 0.90 & 0.87 & 0.84 & 0.88 & 0.78 & 0.76 & 0.79 & 0.87 \\
\hline$\left(\chi_{15}^{2}\right)^{2}$ & 0.41 & 0.42 & 0.46 & 0.42 & 0.39 & 0.45 & 0.33 & 0.31 & 0.32 & 0.36 \\
\hline$N(0,1) * t_{5}$ & 0.32 & 0.28 & 0.29 & 0.32 & 0.25 & 0.28 & 0.30 & 0.24 & 0.25 & 0.17 \\
\hline$N(0,1) * \operatorname{Beta}(1,1)$ & 0.01 & 0.12 & 0.05 & 0.02 & 0.20 & 0.17 & 0.07 & 0.25 & 0.20 & $0.32 \dagger$ \\
\hline$N(0,1) * \exp (1)$ & 0.88 & 0.87 & 0.91 & 0.88 & 0.86 & 0.90 & 0.81 & 0.79 & 0.80 & $0.92 \dagger$ \\
\hline$N(0,1) * \chi_{5}^{2}$ & 0.54 & 0.53 & 0.58 & 0.56 & 0.51 & 0.56 & 0.43 & 0.41 & 0.45 & 0.52 \\
\hline $\operatorname{NMIX}_{2}(0.5,4,0,0)$ & 0.01 & 0.31 & 0.15 & 0.07 & 0.42 & 0.36 & 0.15 & 0.46 & 0.37 & $1.00 \dagger$ \\
\hline $\operatorname{NMIX}_{2}(0.5,0,0,0.9)$ & 0.28 & 0.25 & 0.25 & 0.29 & 0.20 & 0.21 & 0.28 & 0.18 & 0.19 & $0.32 \dagger$ \\
\hline $\operatorname{NMIX}_{2}(0.5,4,0,0.9)$ & 0.90 & 0.91 & 0.94 & 0.90 & 0.88 & 0.93 & 0.71 & 0.69 & 0.79 & $1.00 \dagger$ \\
\hline $\mathrm{NMIX}_{2}(0.9,4,0,0)$ & 0.97 & 0.98 & 0.99 & 0.98 & 0.97 & 0.98 & 0.94 & 0.93 & 0.95 & 0.95 \\
\hline $\operatorname{NMIX}_{2}(0.9,0,0,0.9)$ & 0.08 & 0.06 & 0.10 & 0.09 & 0.04 & 0.09 & 0.08 & 0.03 & 0.06 & 0.05 \\
\hline $\operatorname{NMIX}_{2}(0.9,4,0,0.9)$ & 0.98 & 0.98 & 0.99 & 0.99 & 0.98 & 0.98 & 0.97 & 0.96 & 0.98 & 0.94 \\
\hline
\end{tabular}

Table 4: power comparison of the statistics ( $\alpha=0.05, p=5, n=20$ )

\begin{tabular}{|c|c|c|c|c|c|c|c|c|c|c|}
\hline Alternative & $M N$ & $M W$ & $M E$ & $N N$ & $N W$ & $N E$ & $W N$ & $W W$ & $W E$ & $H Z$ \\
\hline$N(0,1)^{5}$ & 0.05 & 0.06 & 0.05 & 0.05 & 0.05 & 0.05 & 0.05 & 0.05 & 0.05 & 0.04 \\
\hline Cauchy $(0,1)^{5}$ & 1.00 & 1.00 & 1.00 & 1.00 & 0.99 & 0.99 & 1.00 & 0.99 & 0.99 & 0.99 \\
\hline $\operatorname{Logistic}(0,1)^{5}$ & 0.18 & 0.18 & 0.18 & 0.12 & 0.06 & 0.07 & 0.10 & 0.05 & 0.05 & 0.08 \\
\hline$\left(t_{2}\right)^{5}$ & 0.88 & 0.87 & 0.87 & 0.84 & 0.75 & 0.78 & 0.83 & 0.71 & 0.71 & 0.69 \\
\hline$\left(t_{5}\right)^{5}$ & 0.34 & 0.33 & 0.32 & 0.26 & 0.15 & 0.16 & 0.22 & 0.12 & 0.12 & 0.14 \\
\hline $\operatorname{Beta}(1,1)^{5}$ & 0.00 & 0.02 & 0.00 & 0.22 & 0.31 & 0.29 & 0.22 & 0.33 & 0.27 & 0.10 \\
\hline $\operatorname{Beta}(2,2)^{5}$ & 0.00 & 0.01 & 0.01 & 0.12 & 0.17 & 0.16 & 0.12 & 0.18 & 0.15 & 0.06 \\
\hline $\operatorname{Beta}(1,2)^{5}$ & 0.03 & 0.03 & 0.03 & 0.05 & 0.09 & 0.08 & 0.06 & 0.10 & 0.07 & $0.18 \dagger$ \\
\hline $\exp (1)^{5}$ & 0.79 & 0.80 & 0.80 & 0.64 & 0.52 & 0.58 & 0.58 & 0.48 & 0.49 & $0.81 \dagger$ \\
\hline $\operatorname{Lognormal}(0,0.5)^{5}$ & 0.60 & 0.58 & 0.60 & 0.44 & 0.32 & 0.39 & 0.38 & 0.27 & 0.29 & 0.49 \\
\hline $\operatorname{Gamma}(0.5,1)^{5}$ & 0.97 & 0.97 & 0.97 & 0.91 & 0.87 & 0.91 & 0.88 & 0.84 & 0.85 & $0.99 \dagger$ \\
\hline $\operatorname{Gamma}(5,1)^{5}$ & 0.21 & 0.20 & 0.20 & 0.13 & 0.08 & 0.09 & 0.10 & 0.06 & 0.06 & 0.17 \\
\hline$\left(\chi_{5}^{2}\right)^{5}$ & 0.39 & 0.40 & 0.40 & 0.25 & 0.16 & 0.20 & 0.21 & 0.13 & 0.13 & 0.34 \\
\hline$\left(\chi_{15}^{2}\right)^{5}$ & 0.15 & 0.14 & 0.15 & 0.09 & 0.05 & 0.06 & 0.08 & 0.05 & 0.04 & 0.12 \\
\hline$N(0,1)^{4} * t_{5}$ & 0.10 & 0.09 & 0.10 & 0.07 & 0.05 & 0.06 & 0.07 & 0.04 & 0.05 & 0.06 \\
\hline$N(0,1)^{4} * \operatorname{Beta}(1,1)$ & 0.03 & 0.03 & 0.03 & 0.06 & 0.07 & 0.07 & 0.06 & 0.07 & 0.07 & 0.06 \\
\hline$N(0,1)^{4} * \exp (1)$ & 0.18 & 0.19 & 0.19 & 0.12 & 0.07 & 0.08 & 0.09 & 0.06 & 0.05 & 0.13 \\
\hline$N(0,1)^{4} * \chi_{5}^{2}$ & 0.11 & 0.10 & 0.10 & 0.07 & 0.04 & 0.05 & 0.06 & 0.05 & 0.04 & 0.08 \\
\hline $\operatorname{NMIX}_{5}(0.5,4,0,0)$ & 0.03 & 0.03 & 0.03 & 0.07 & 0.09 & 0.09 & 0.06 & 0.10 & 0.08 & $0.17 \dagger$ \\
\hline $\operatorname{NMIX}_{5}(0.5,0,0,0.9)$ & 0.65 & 0.61 & 0.63 & 0.59 & 0.37 & 0.41 & 0.52 & 0.30 & 0.30 & 0.54 \\
\hline $\operatorname{NMIX}_{5}(0.5,4,0,0.9)$ & 0.82 & 0.82 & 0.83 & 0.73 & 0.59 & 0.65 & 0.69 & 0.54 & 0.54 & $0.96 \dagger$ \\
\hline $\operatorname{NMIX}_{5}(0.9,4,0,0)$ & 0.40 & 0.40 & 0.43 & 0.30 & 0.20 & 0.24 & 0.26 & 0.15 & 0.16 & 0.32 \\
\hline $\operatorname{NMIX}_{5}(0.9,0,0,0.9)$ & 0.10 & 0.10 & 0.10 & 0.08 & 0.04 & 0.05 & 0.06 & 0.04 & 0.04 & 0.07 \\
\hline $\operatorname{NMIX}_{5}(0.9,4,0,0.9)$ & 0.43 & 0.42 & 0.45 & 0.26 & 0.20 & 0.22 & 0.23 & 0.16 & 0.17 & 0.40 \\
\hline
\end{tabular}


Table 5: power comparison of the statistics ( $\alpha=0.05, p=5, n=50)$

\begin{tabular}{|c|c|c|c|c|c|c|c|c|c|c|}
\hline Alternative & $M N$ & $M W$ & $M E$ & $N N$ & $N W$ & $N E$ & $W N$ & $W W$ & $W E$ & $H Z$ \\
\hline$N(0,1)^{5}$ & 0.04 & 0.04 & 0.05 & 0.05 & 0.05 & 0.05 & 0.04 & 0.04 & 0.06 & 0.04 \\
\hline Cauchy $(0,1)^{5}$ & 1.00 & 1.00 & 1.00 & 1.00 & 1.00 & 1.00 & 1.00 & 1.00 & 1.00 & $1.00 \dagger$ \\
\hline $\operatorname{Logistic}(0,1)^{5}$ & 0.41 & 0.37 & 0.37 & 0.37 & 0.28 & 0.32 & 0.32 & 0.26 & 0.26 & 0.14 \\
\hline$\left(t_{2}\right)^{5}$ & 1.00 & 0.99 & 1.00 & 1.00 & 1.00 & 1.00 & 1.00 & 1.00 & 1.00 & 1.00 \\
\hline$\left(t_{5}\right)^{5}$ & 0.68 & 0.67 & 0.70 & 0.72 & 0.65 & 0.67 & 0.69 & 0.61 & 0.62 & 0.30 \\
\hline $\operatorname{Beta}(1,1)^{5}$ & 0.00 & 0.08 & 0.00 & 0.68 & 0.86 & 0.83 & 0.66 & 0.85 & 0.82 & 0.50 \\
\hline $\operatorname{Beta}(2,2)^{5}$ & 0.00 & 0.00 & 0.00 & 0.27 & 0.44 & 0.42 & 0.27 & 0.44 & 0.40 & 0.14 \\
\hline $\operatorname{Beta}(1,2)^{5}$ & 0.05 & 0.06 & 0.07 & 0.04 & 0.14 & 0.11 & 0.03 & 0.11 & 0.08 & $0.66 \dagger$ \\
\hline $\exp (1)^{5}$ & 1.00 & 1.00 & 1.00 & 1.00 & 1.00 & 1.00 & 1.00 & 1.00 & 1.00 & $1.00 \dagger$ \\
\hline $\operatorname{Lognormal}(0,0.5)^{5}$ & 0.99 & 0.99 & 0.99 & 0.99 & 0.98 & 0.99 & 0.96 & 0.97 & 0.97 & 0.96 \\
\hline $\operatorname{Gamma}(0.5,1)^{5}$ & 1.00 & 1.00 & 1.00 & 1.00 & 1.00 & 1.00 & 1.00 & 1.00 & 1.00 & $1.00 \dagger$ \\
\hline $\operatorname{Gamma}(5,1)^{5}$ & 0.67 & 0.69 & 0.70 & 0.56 & 0.54 & 0.55 & 0.48 & 0.48 & 0.51 & 0.47 \\
\hline$\left(\chi_{5}^{2}\right)^{5}$ & 0.95 & 0.95 & 0.95 & 0.90 & 0.89 & 0.92 & 0.85 & 0.84 & 0.87 & 0.88 \\
\hline$\left(\chi_{15}^{2}\right)^{5}$ & 0.49 & 0.50 & 0.50 & 0.35 & 0.36 & 0.36 & 0.31 & 0.27 & 0.30 & 0.28 \\
\hline$N(0,1)^{4} * t_{5}$ & 0.20 & 0.19 & 0.18 & 0.16 & 0.13 & 0.15 & 0.14 & 0.13 & 0.12 & 0.09 \\
\hline$N(0,1)^{4} * \operatorname{Beta}(1,1)$ & 0.02 & 0.03 & 0.02 & 0.04 & 0.08 & 0.07 & 0.06 & 0.09 & 0.08 & $0.10 \dagger$ \\
\hline$N(0,1)^{4} * \exp (1)$ & 0.55 & 0.59 & 0.57 & 0.44 & 0.43 & 0.44 & 0.38 & 0.36 & 0.41 & 0.39 \\
\hline$N(0,1)^{4} * \chi_{5}^{2}$ & 0.25 & 0.29 & 0.27 & 0.20 & 0.19 & 0.20 & 0.18 & 0.14 & 0.16 & 0.16 \\
\hline $\operatorname{NMIX}_{5}(0.5,4,0,0)$ & 0.03 & 0.02 & 0.03 & 0.06 & 0.12 & 0.11 & 0.07 & 0.14 & 0.14 & $0.70 \dagger$ \\
\hline $\operatorname{NMIX}_{5}(0.5,0,0,0.9)$ & 0.90 & 0.88 & 0.84 & 0.93 & 0.87 & 0.86 & 0.94 & 0.84 & 0.83 & $0.98 \dagger$ \\
\hline $\operatorname{NMIX}_{5}(0.5,4,0,0.9)$ & 1.00 & 1.00 & 1.00 & 1.00 & 1.00 & 1.00 & 1.00 & 1.00 & 1.00 & $1.00 \dagger$ \\
\hline $\operatorname{NMIX}_{5}(0.9,4,0,0)$ & 0.88 & 0.89 & 0.88 & 0.79 & 0.79 & 0.81 & 0.74 & 0.74 & 0.76 & $0.92 \dagger$ \\
\hline $\operatorname{NMIX}_{5}(0.9,0,0,0.9)$ & 0.17 & 0.15 & 0.13 & 0.13 & 0.09 & 0.12 & 0.13 & 0.09 & 0.09 & 0.11 \\
\hline $\operatorname{NMIX}_{5}(0.9,4,0,0.9)$ & 0.99 & 0.99 & 0.99 & 0.96 & 0.95 & 0.96 & 0.89 & 0.88 & 0.91 & 0.91 \\
\hline
\end{tabular}

best power against some alternatives with skewed marginals and some normal mixtures. However the power against symmetric shorter tailed marginals is not good. Apparently the statistics $M N, M W$, $M E$ and $H Z$ show very similar phenomena in power. For the alternatives $N(0,1)^{p-1} * F_{2}$, when $F_{2}$ has a symmetric thicker tailed, a symmetric shorter tailed, or a skewed distribution, we can see almost the same things as above.

Let us compare the normal approximation and the Enomoto transformation to the kurtosis. When we compare the statistics $N N$ and $N E$ with the normal approximation to the skewness, or $W N$ and $W E$ with Wilson-Hilferty to the skewness, $N N, W N$ show better power than $N E, W E$ against alternatives with symmetric thicker tailed marginals. Conversely, $N E, W E$ have much better power against alternatives with symmetric shorter tailed marginals. They show similar power against skewed marginal alternatives. The statistics $M N$ and $M E$ have comparable power. $M E$ shows better power for $p=2$ and they have almost the same power for $p=5$. However $M N$ and $M E$ show terribly low power against alternatives with symmetric shorter tailed marginals.

As an omnibus test statistic, we recommend the statistic $N E$ or $W E$. The other statistics could show better power than these statistics in some special alternatives; however, $N E$ or $W E$ has relatively good power against almost all alternatives considered, and the null distribution is well described by the $\chi^{2}$-distribution. Comparing the two statistics, the power of $N E$ is better than WE. The Enomoto's transformation to the kurtosis may be replace by the normal approximation to the kurtosis.

\section{Conclusions}

In this paper, we considered omnibus tests for multivariate normality based on the Mardia's multivariate skewness and kurtosis. The idea is to use the normalizing transformations for the multivariate skewness and kurtosis. We used the normal approximation using mean and variance, the Wilson- 
Hilferty transformation of a $\chi^{2}$-variable, and the transformation proposed by Enomoto et al. (2019) for the Mardia's kurtosis.

We investigated the power of the tests under several alternatives. The sum of the statistics of the Mardia's skewness and the transformations of the Mardia's kurtosis have good power against alternatives with skewed marginals. The normal approximation to the Mardia's kurtosis shows good power against alternatives with symmetric thicker tailed marginals and the Wilson-Hilferty transformation to the Mardia's kurtosis shows good power against alternatives with symmetric shorter tailed marginals. No tests have best power in all kinds of alternatives; however, the statistic $N E$ should be recommended as an omnibus statistic because of the relatively good power in many of the alternatives considered. It has the normal approximation to the Mardia's skewness and the Enomoto's normalizing transformation to the Mardia's kurtosis.

\section{Acknowledgement}

This work was supported by 2020 Hongik University Research Fund.

\section{References}

Anscombe FJ and Glynn WJ (1983). Distribution of the kurtosis statistic $b_{2}$ for normal statistics, Biometrika, 70, 227-234.

Baringhaus L and Henze N (1992). Limit distributions for Mardia's measure of multivariate skewness, Annals of Statistics, 20, 1889-1902.

Bowman KO and Shenton LR (1975). Omnibus test contours for departures from normality based on $\sqrt{b_{1}}$ and $b_{2}$, Biometrika, 62, 243-250.

D’Agostino RB (1970). Transformation to normality of the null distribution of $g_{1}$, Biometrika, 57, 679-681.

D'Agostino RB and Pearson ES (1973). Tests for departure from normality: Empirical results for the distributions of $b_{2}$ and $\sqrt{b_{1}}$, Biometrika, 60, 613-622.

D'Agostino RB and Pearson ES (1974). Correction and amendment: Tests for departure from normality: Empirical results for the distributions of $b_{2}$ and $\sqrt{b_{1}}$, Biometrika, 61, 647.

Doornik JA and Hansen H (2008). An omnibus test for univariate and multivariate normality, Oxford Bulletin of Economics and Statistics, 70, 927-939.

Enomoto R, Hanusz Z, Hara A, and Seo T (2019). Multivariate normality test using normalizing transformation for Mardia's multivariate kurtosis, Communications in Statistics-Simulation and Computation, 49, 684-698.

Farrell PJ, Salibian-Barrera M, and Naczk K (2007). On tests for multivariate normality and associated simulation studies, Journal of Statistical Computation and Simulation, 77, 1065-1080.

Gel YR and Gastwirth JL (2008). A robust modification of the Jarque-Bera test of normality, Economics Letters, 99, 30-32.

Hanusz Z, Enomoto R, Seo T, and Koizumi K (2018). A Monte Carlo comparison of Jarque-Bera type tests and Henze-Zirkler test of multivariate normality, Communications in Statistics - Simulation and Computation, 47, 1439-1452.

Henze N (1994). On Mardia's kurtosis for multivariate normality, Communications in StatisticsTheory and Methods, 23, 1031-1045.

Henze N (2002). Invariant tests for multivariate normality: A critical review, Statistical Papers, 43, 467-506.

Henze N and Zirkler B (1990). A class of invariant consistent tests for multivariate normality, Com- 
munications in Statistics-Theory and Methods, 19, 3539-3617.

Horswell RL and Looney SW (1992). A comparison of tests for multivariate normality that are based on measures of multivariate skewness and kurtosis, Journal of Statistical Computation and Simulation, 42, 21-38.

Jarque C and Bera A (1980). Efficient tests for normality, homoscedasticity and serial independence of regression residuals, Economics Letters, 6, 255-259.

Kim N (2016). A robustified Jarque-Bera test for multivariate normality, Economics Letters, 140, 48-

Konishi S (1981). Normalizing transformations of some statistics in multivariate analysis, Biometrika, 68, 647-651.

Mardia KV (1970). Measures of multivariate skewness and kurtosis with applications, Biometrika, 57, 519-530.

Mardia KV (1974). Applications of some measures of multivariate skewness and kurtosis for testing normality and robustness studies, Sankhya A, 36, 115-128.

Mardia KV (1975). Assessment of multinormality and the robustness of Hotelling's $T^{2}$ test Applied Statistics, 24, 163-171.

Mardia KV and Foster K (1983). Omnibus tests of multinormality based on skewness and kurtosis, Communications of Statistics-Theory and Methods, 12, 207-221.

Mardia KV and Kanazawa M (1983). The null distribution of multivariate kurtosis, Communications of Statistics-Simulation and Computation, 12, 569-576.

Mecklin CJ and Mundfrom DJ (2005). A Monte Carlo comparison of the Type I and Type II error rates of tests of multivariate normality, Journal of Statistical Computation and Simulation, $\mathbf{7 5}$, 93-107.

Pearson ES (1956). Some aspects of the geometry of statistics, Journal of the Royal Statistical Society, Series A, 119, 125-146.

Rao CR (1948). Test of significance in multivariate analysis, Biometrika, 35, 58-79.

Romeu JL and Ozturk A (1993). A comparative study of goodness-of-fit tests for multivariate normality, Journal of Multivariate Analysis, 46, 309-334.

Seo T, Kanda T, and Fujikoshi Y (1994). The effects on the distributions of sample canonical correlations under nonnormality, Communications in Statistics - Theory and Methods, 23, 2615-2628.

Shenton LR and Bowman KO (1977). A bivariate model for the distribution of $\sqrt{b_{1}}$ and $b_{2}$, Journal of the American Statistical Association, 72, 206-211.

Srivastava MS and Hui TK (1987). On assessing multivariate normality based on Shapiro-Wilk $W$ statistic, Statistics \& Probability Letters, 5, 15-18.

Srivastava DK and Mudholkar GS (2003). Goodness of fit tests for univariate and multivariate normal models. In Khattree R., and Rao, C. R., eds., Handbook of Statistics 22: Statistics in Industry, Elsevier, North Holland, 869-906.

Stehlík M, Fabián Z, and Střelec L (2012). Small sample robust testing for normality against Pareto tails, Communications in Statistics - Simulation and Computation, 41, 1167-1194.

Thode Jr. HC (2002). Testing for Normality, Marcel Dekker, New York.

Urzua CM (1996). On the correct use of omnibus tests for normality, Economics Letters, 53, 247-251. 